\title{
Erratum
}

\section{Midlife moderation-quantified healthy diet and 40-year mortality risk from CHD: the prospective National Heart, Lung, and Blood Institute Twin Study - ERRATUM}

\author{
Jun Dai Ruth E. Krasnow and T. Reed
}

(First published online 18 May 2016)

doi: $10.1017 /$ S0007114516001914

There are minor errors in Table 1 on page 4.

Original text: Food groups. In the twelfth row entry, first column, Table 1.

Revised text: Sweets

Original text: Food items. In the twelfth row entry, second column, Table 1

Revised text: candy, sugar, chocolate, jam, jelly, syrup, desserts

Corrected Table 1 as follows:

Table 1. The reference value for the maximal score of 10 for each component of moderation-quantified healthy diet (MQHD) score and alternative moderation-quantified healthy diet (aMQHD) score among 910 twins $^{\star}$

(Medians and interquartile ranges (IQR))

\begin{tabular}{|c|c|c|c|c|c|}
\hline \multirow[b]{2}{*}{ Food groups } & \multirow{2}{*}{\multicolumn{3}{|c|}{$\begin{array}{l}\text { Reference value for the } \\
\text { maximal score of } 10\end{array}$}} & \multicolumn{2}{|r|}{ Intake } \\
\hline & & & & Median & IQR \\
\hline \multicolumn{6}{|c|}{ Individual score components common to both MQHD score and aMQHD score } \\
\hline & Servin & gs/d & & Servings/d & \\
\hline Grains & $\begin{array}{l}\text { Spaghetti/pasta, rice, cereal, bread, sweet roll/ } \\
\text { donut, cake/pie/pastry }\end{array}$ & & & 6 & $4-7$ \\
\hline Fruits & Fruits, fruit juice & & & 2 & $1-3$ \\
\hline Vegetables & Salad, cooked vegetables & & & 2.5 & $2-3$ \\
\hline Dairy products & See footnote† & & & $\begin{array}{l}4.5 \dagger \text { or } \\
2.0 \ddagger\end{array}$ & $\begin{array}{l}3.1-6.0 \dagger \text { or } 1.2- \\
3.0 \ddagger\end{array}$ \\
\hline \multirow[t]{2}{*}{ Alcohol } & $\begin{array}{l}\text { Beer, wine, other alcoholic beverages (spirit, } \\
\text { cocktail) }\end{array}$ & & & 0.6 & $0-1.6$ \\
\hline & & $\begin{array}{l}\text { Servings/ } \\
\text { week }\end{array}$ & $\begin{array}{l}\text { Servings/ } \\
\quad \mathrm{d}\end{array}$ & Servings/w & leek \\
\hline Fish and shellfish & Shellfish, non-shellfish & 4 & 0.57 & 1.5 & $0-3$ \\
\hline Poultry & Chicken/turkey, fried chicken & 2 & 0.29 & 2 & $1-3$ \\
\hline Red meat & $\begin{array}{l}\text { Pork, ham, bacon, sausage, steak, pot roast, beef, hamburger, luncheon } \\
\text { meat, hotdog, liver, sweetbreads, brain, fried meat }\end{array}$ & 3 & 0.43 & 14 & $11-18$ \\
\hline Eggs & Eggs, fried egg & 3 & 0.43 & 6 & $3-11$ \\
\hline Nuts & Peanut butter, nuts & 4 & 0.57 & 3 & $1-5$ \\
\hline $\begin{array}{l}\text { Potatoes and maize } \\
\text { chips }\end{array}$ & Potatoes, fried potatoes, potato chips, maize chips & 3 & 0.43 & 9 & $5-12$ \\
\hline Sweets & Candy, sugar, chocolate, jam, jelly, syrup, desserts & 3 & 0.43 & 19 & $12-17$ \\
\hline & - & 4 & 0.57 & 1.56 & $1.39-1.74$ \\
\hline
\end{tabular}


Table 1. Continued

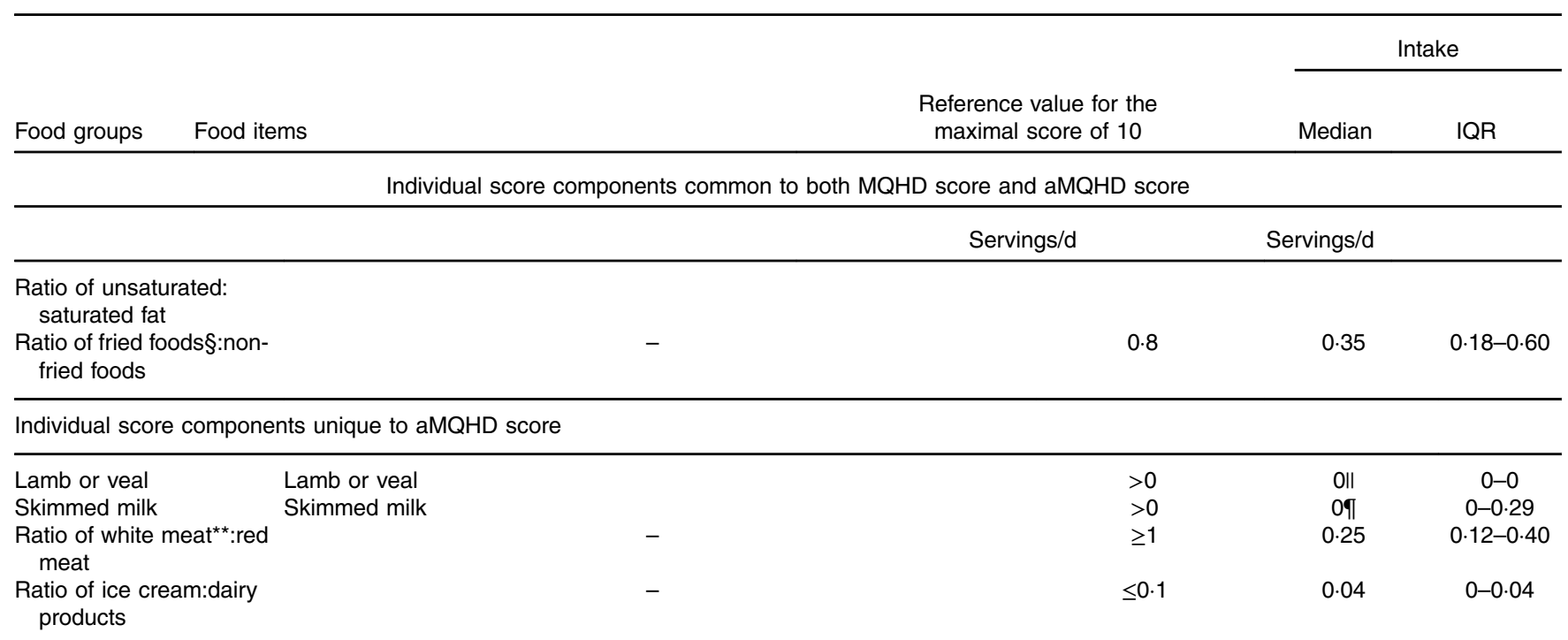

* Servings were based on daily $10460 \mathrm{~kJ}(2500 \mathrm{kcal})$.

† Dairy products referred to all milk and milk products including whole milk, skimmed milk, chocolate milk, ice cream, milk-containing products such as pudding, butter and cheese.

‡ Dairy products the same as defined above with exclusion of butter.

$\S$ Fried food included fried potatoes, potato chips, fried eggs, fried meat, fried chicken, fried fish and fried shellfish.

II Servings/week.

II Servings/d.

** White meat includes seafood, fish and poultry.

\section{Reference}

Dai J, Krasnow RE \& Reed T (2016) Midlife moderation-quantified healthy diet and 40-year mortality risk from CHD: the prospective National Heart, Lung, and Blood Institute Twin Study. Br J Nutr 116, 326-334. 